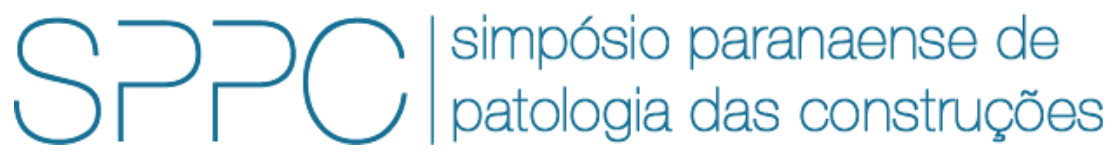

ISSN 2526-7248 artigo 2SPPC1023, pp. 275-288, 2017

\title{
Avaliação de testemunhos de concreto compactado com rolo, com indícios de ataque por sulfato de origem interna, pelo ensaio de permeabilidade ao ar - método Torrent
}

\author{
Betina Lepretti Medeiros ${ }^{1}$, Bruna Slomisky2, Bruno Endo Ribeiro ${ }^{3}$, Mariana O.G.P. Bragança ${ }^{4}$, Kleber \\ Franke Portella ${ }^{5}$, Marcelo Henrique Farias de Medeiros ${ }^{6}$, Isabela Cristina de Oliveira ${ }^{7}$ \\ 1'Doutoranda, MSc., Institutos Lactec, betina@lactec.org.br \\ 2 Graduanda, Institutos Lactec, bruna.slominsky@lactec.org.br \\ ${ }^{3}$ Graduando, Institutos Lactec, bruno.ribeiro@lactec.org.br \\ ${ }^{4}$ Professora Doutora, Institutos Lactec, mariana.portella@lactec.org.br \\ ${ }^{5}$ Professor Doutor, Institutos Lactec, portella@lactec.org.br \\ ${ }^{6}$ Professor Doutor, Universidade Federal do Paraná (UFPR), medeiros.ufpr@gmail.com \\ ${ }^{7}$ Engenheira Civil MSc., Companhia Paranaense de Energia (Copel), isabela.oliveira@copel.com
}

Resumo: Agentes agressivos são conduzidos ao interior do concreto pelo fenômeno de transporte de fluidos, através da porosidade sob condições de pressão entre o material e o meio externo. Reações com as substâncias químicas provenientes do meio externo ou como subprodutos de reações dos componentes do concreto, tais como o ataque de sulfato de origem interna, acontecem na presença de água contida nos poros. A absorção pelo concreto depende da porosidade devido à interconexão dos poros capilares, principalmente do tamanho dos poros. A permeabilidade do concreto é considerada como um parâmetro de referência para as análises de durabilidade de estruturas de concreto. Um método não destrutivo válido para a avaliação da permeabilidade de concretos é o proposto por Torrent, visando avaliar e classificar a qualidade do concreto. No presente trabalho, o objetivo foi avaliar a permeabilidade, pelo método de Torrent, de testemunhos de concreto compactado com rolo (CCR) com indícios de ataque por sulfato interno, extraídos de uma barragem de usina hidrelétrica. Os resultados obtidos com este método de ensaio permitiram avaliar a qualidade do CCR como normal e ruim, com valores de $\mathrm{kT}$ entre 1,04E-15 e 3,29E-17m². Este trabalho foi desenvolvido no âmbito do Programa de P\&D ANEEL, projeto 6491-0301/2013.

Palavras-chave: Concreto compactado com rolo, ataque por sulfato de origem interna, permeabilidade ao ar.

\begin{abstract}
Aggressive agents are conducted to the interior of the concrete by fluid transport phenomena, through porosity under pressure conditions between the material and the external environment. Reactions with the chemicals products from the external environment or as byproducts of the reactions between concrete components, such as the attack of sulfate of internal origin, occur in the presence of pores water. The absorption by the concrete, depending on the porosity due to the interconnection of the capillary pores, mainly by the size of the pores. The permeability of the concrete is considered as a reference parameter for the analysis of the durability of concrete structures. A valid non-destructive method to evaluate the permeability of concrete is proposed by Torrent, to evaluate and classify the quality of the concrete. The objective of the present work was to evaluate the permeability, by Torrent method, of compacted roll (RCC) concrete samples with evidence of internal sulfate attack. The results obtained with this test method allowed to evaluate the CCR quality as normal and poor, with values of kT between $1.04 \mathrm{E}-15$ and $3.29 \mathrm{E}-17 \mathrm{~m}^{2}$.
\end{abstract}

Keywords: Roll compacted concrete (RCC), internal sulfate attack; air permeability. 
MEDEIROS, B.L.; ET AL., AVALIAÇÃO DE TESTEMUNHOS DE CONCRETO COMPACTADO COM ROLO, COM INDÍCIOS DE ATAQUE POR SULFATO DE ORIGEM INTERNA, PELO ENSAIO DE PERMEABILIDADE AO AR - MÉTODO TORRENT. $2^{\circ}$ Simpósio Paranaense de Patologia das Construções (20 SPPC), 2SPPC1023, pp. 275-288, 2017. DOI: 10.4322/2SPPC.2017.023

\section{Introdução}

Durante a vida útil de uma estrutura de concreto pode ser percebida a degradação e, consequentemente, a diminuição da durabilidade pela ocorrência de manifestações patológicas. Segundo Campos [1], o surgimento de processos expansivos em concretos pode ser induzido por reações químicas, como a reação álcali agregado (RAA), o ataque por sulfato externo (ASE) e o ataque por sulfato interno (ASI) que provocam mudanças de volume na microestrutura, iniciando, desta forma, uma microfissuração como primeira manifestação patológica e possibilitando assim a entrada de novos agentes agressivos.

Agentes agressivos são introduzidos nas estruturas de concreto de cimento Portland a partir de ambientes externos, ou até mesmo de seus insumos, como aqueles presentes na composição química do cimento, nos agregados ou na água de amassamento [2]. Especificamente, os íons sulfato podem penetrar no interior das estruturas de concreto por redes capilares, dissolvidos na água, ou estar presentes em insumos, deteriorando quimicamente o material, devido ao desenvolvimento de subprodutos expansivos no interior dos poros.

Segundo descrito por Melo [2], as manifestações patológicas decorrentes do ataque por sulfatos internos são, normalmente, apresentadas em forma de fissuração devido à expansão, à diminuição progressiva da resistência e à perda de massa do concreto.

Dentre os principais tipos de ASI, Campos [1] expõe em seu trabalho que a presença de altos teores de sulfatos no cimento, em conjunto com as altas temperaturas de sua hidratação ou mesmo proveniente do ambiente, proporciona a formação de etringita tardia (DEF).

Outro tipo de ASI é o que se manifesta quando a composição do concreto, agregados com presença de sulfatos de ferro na forma de pirita ou pirrotita. Estes agregados, durante sua reação primária (oxidação), causam expansão, gerando sulfatos que em conjunto com os aluminatos do cimento (reação secundária) formam etringita associada de maior magnitude que a obtida na primeira reação [1].

Exemplos de ASI decorrentes de uso de agregados com alto teor de sulfato de ferro foram verificados nas barragens de Graus, Tavascan, Rumedo e Paso Nuevo localizadas na Espanha [1]. Nestas estruturas, diversos estudos de campo vêm sendo realizados, mas há pouca divulgação bibliográfica, relativa aos seus resultados.

A avaliação das condições do concreto das estruturas das barragens durante a sua operação é importante, sob o ponto de vista de segurança. Assim, como exemplo, na barragem Sella Zerbino, na Itália, foi inferido como uma possível causa do colapso ocorrido um concreto com resistência inadequada [3]. A deterioração do material de outras estruturas de barragem foi associada por Zuffo [4] como resultante do emprego de agregados reativos ou de baixa resistência.

O concreto compactado com rolo (CCR) aplicado em barragens contém em sua composição agregados de diâmetro máximo de até $50 \mathrm{~mm}$, possui um baixo consumo de cimento, e na sua produção são utilizados equipamentos para o 
MEDEIROS, B.L.; ET AL., AVALIAÇÃO DE TESTEMUNHOS DE CONCRETO COMPACTADO COM ROLO, COM INDÍCIOS DE ATAQUE POR SULFATO DE ORIGEM INTERNA, PELO ENSAIO DE PERMEABILIDADE AO AR - MÉTODO TORRENT. $2^{\circ}$ Simpósio

Paranaense de Patologia das Construções (20 SPPC), 2SPPC1023, pp. 275-288, 2017. DOI: 10.4322/2SPPC.2017.023

lançamento e compactação de grandes volumes. Esta técnica é usualmente utilizada para a confecção de barragens de reservatórios para armazenamento de água, amortização de cheias e geração de energia elétrica [5]. Marques Filho [5] comentou que as juntas entre camadas de lançamento sucessivas são de fundamental importância, pois estão diretamente relacionadas à resistência mecânica e à permeabilidade pelo maciço.

O concreto é um material naturalmente poroso, pela utilização de água para dar o início das reações, pela incorporação de ar durante sua fabricação, bem como pela retração durante a cura [5].

Sahuinco [6] citou que os principais mecanismos de transporte de fluidos com ou sem agentes agressivos acontece pelos poros da pasta, a partir dos processos de capilaridade (absorção), gradiente de pressão (permeabilidade), gradiente de concentração (difusão) e devido à aplicação de um campo elétrico (migração).

A permeabilidade do concreto pode ser determinada a partir de ensaios normatizados como o da permeabilidade à agua, a absorção por capilaridade e por imersão [7-9]. Outro método utilizado é o não destrutivo para a avaliação da permeabilidade ao ar idealizado por Torrent [10]. Com esta metodologia de ensaio se mede, indiretamente, a porosidade do concreto de uma camada superficial [11], possibilitando realizar correlações entre os indicadores de durabilidade, como no estudo realizado por Ebensperger e Torrent [12], onde foram avaliados o ataque por cloretos e o coeficiente de permeabilidade ao ar, denominado como kT. Neves [13], fez análises correlacionando o estudo de kT com o fator água/cimento, a sua resistência mecânica e a carbonatação.

O método desenvolvido por Torrent [10] consiste no uso de uma câmara de célula dupla, onde se produz uma pressão negativa sob a superfície do material com o auxílio de uma bomba a vácuo (Figura 1). A partir da aplicação deste método não destrutivo, se obtém dados do coeficiente de permeabilidade (kT), da espessura da camada onde se tem a leitura $(\mathrm{L})$, da pressão $(\mathrm{Pi})$ e do tempo de leitura em segundos [14].

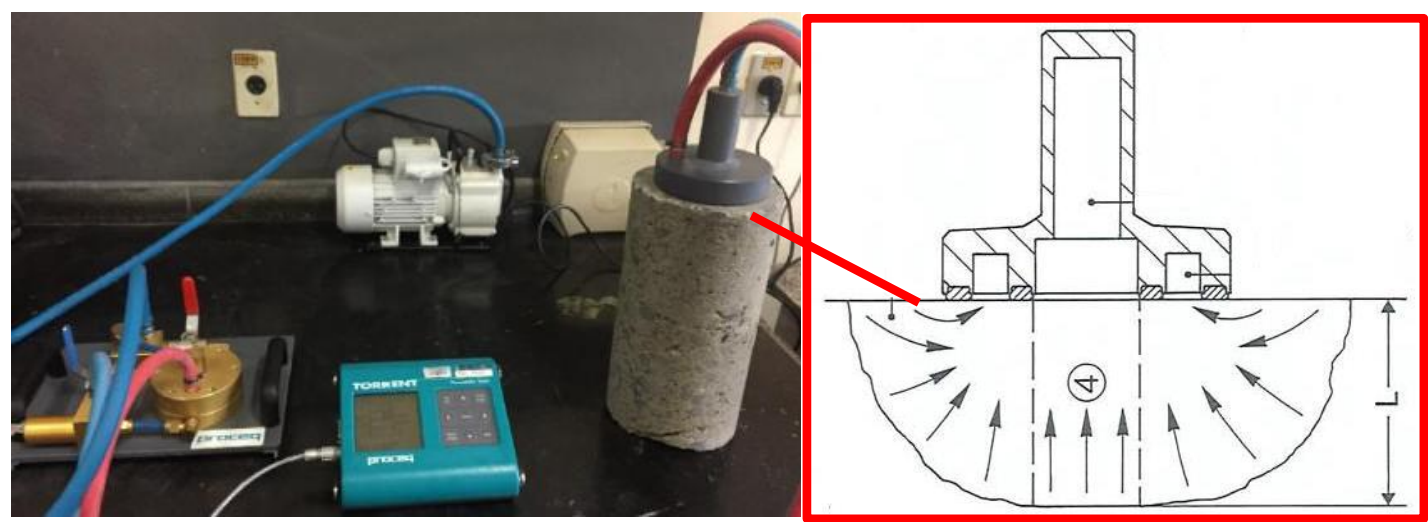

Figura 1: Foto ilustrativa dos instrumentos utilizados na leitura de $k T$ e $L$ realizada em testemunhos de CCR, pelo método de Torrent.

O autor do método Torrent [10], considera que a porosidade de um corpo de prova não muda ao longo da extensão e que podem ser realizadas avaliações de estruturas em corpos de provas ou in situ, como estruturas de pontes estudadas em 
MEDEIROS, B.L.; ET AL., AVALIAÇÃO DE TESTEMUNHOS DE CONCRETO COMPACTADO COM ROLO, COM INDÍCIOS DE ATAQUE POR SULFATO DE ORIGEM INTERNA, PELO ENSAIO DE PERMEABILIDADE AO AR - MÉTODO TORRENT. $2^{\circ}$ Simpósio Paranaense de Patologia das Construções (20 SPPC), 2SPPC1023, pp. 275-288, 2017. DOI: 10.4322/2SPPC.2017.023

trabalhos realizados, principalmente, em cobrimentos ou argamassas de revestimento, visando investigar os processos de deterioração ou de corrosão das estruturas, seja pela carbonatação, pela difusão de cloretos ou de outros íons agressivos ao meio. Assim, para avaliar a condição do concreto, e classificar o mesmo pelo método, é necessário obter o valor da resistividade do material a ser ensaiado. Segundo Torrent [10], esta classificação é válida somente para concretos com até 90 dias de idade. Para maiores tempos, as considerações sobre a qualidade do concreto, a partir dos valores de $\mathrm{kT}$, mostradas na tabela de classificação Torrent (Tabela 1), não podem ser aplicadas diretamente, sendo somente considerado o coeficiente de permeabilidade $(\mathrm{kT})$.

Tabela 1: Qualidade de concretos pelo Método Torrent [10].

\begin{tabular}{ccc}
\hline Qualidade de cobertura do concreto & Índice & $\mathbf{k T}\left(\mathbf{1 0}^{-\mathbf{1 6}} \mathbf{m}^{\mathbf{2}}\right)$ \\
\hline Muito ruim & 5 & $>10$ \\
Ruim & 4 & $1,0-10$ \\
Normal & 3 & $0,1-1,0$ \\
Bom & 2 & $0,01-0,1$ \\
Muito bom & 1 & $<0,01$ \\
\hline
\end{tabular}

A determinação da resistividade elétrica é um parâmetro importante para a avaliação da corrosão de estruturas armadas, pois concretos de alta resistividade têm menor chance de desenvolver corrosão [6], servindo o resultado desta técnica como parâmetro na qualificação de concretos pelo método Torrent. Este ensaio consiste em introduzir uma pequena corrente elétrica na superfície no concreto para a obtenção da sua resistividade.

Segundo Jacobs [15], os resultados obtidos com o ensaio de permeabilidade ao ar são influenciados pela composição do concreto, pelos materiais utilizados, pela temperatura e por demais condições, como a ocorrência de fissuras e o revestimento.

Sentone [14] citou em seu trabalho que existem diversos métodos para se avaliar a permeabilidade ao ar em materiais porosos, assim como equipamentos e técnicas diferenciados, podendo ser medida em laboratório ou, mesmo, in situ.

Para a avaliação de obras existentes, como nas barragens de concreto, é possível a realização de extração de testemunhos para avaliação da qualidade da estrutura, em termos de análises mecânicas e físico-química (durabilidade) [5]. Ainda, é possível identificar problemas em materiais decorrentes de processos construtivos das estruturas de CCR.

O objetivo deste estudo foi determinar a permeabilidade ao ar de CCR de testemunhos extraídos de barragens, com o intuito de desenvolver, paralelamente, a análise dos resultados obtidos pela técnica desenvolvida por Torrent. Esta, foi associada a ensaios não destrutivos como de ultrassom e de resistividade, bem como de absorção por imersão e por capilaridade, complementados por microanálises para avaliação das técnicas, em conjunto, na análise dos efeitos provocados pelo ataque por sulfatos de origem interna. 
MEDEIROS, B.L.; ET AL., AVALIAÇÃO DE TESTEMUNHOS DE CONCRETO COMPACTADO COM ROLO, COM INDÍCIOS DE ATAQUE POR SULFATO DE ORIGEM INTERNA, PELO ENSAIO DE PERMEABILIDADE AO AR - MÉTODO TORRENT. $2^{\circ}$ Simpósio Paranaense de Patologia das Construções (20 SPPC), 2SPPC1023, pp. 275-288, 2017. DOI: 10.4322/2SPPC.2017.023

\section{Experimental}

A extração de testemunhos foi realizada na face jusante de uma barragem em CCR, com profundidade de $3,01 \mathrm{~m}$ como está indicado na Figura 2. Os concretos avaliados foram retirados das cotas iniciais, conforme destacado em vermelho na Figura 2, onde as camadas compactadas foram lançadas nos primeiros meses de construção e constituem um volume total de $12.577 \mathrm{~m}^{3}$.

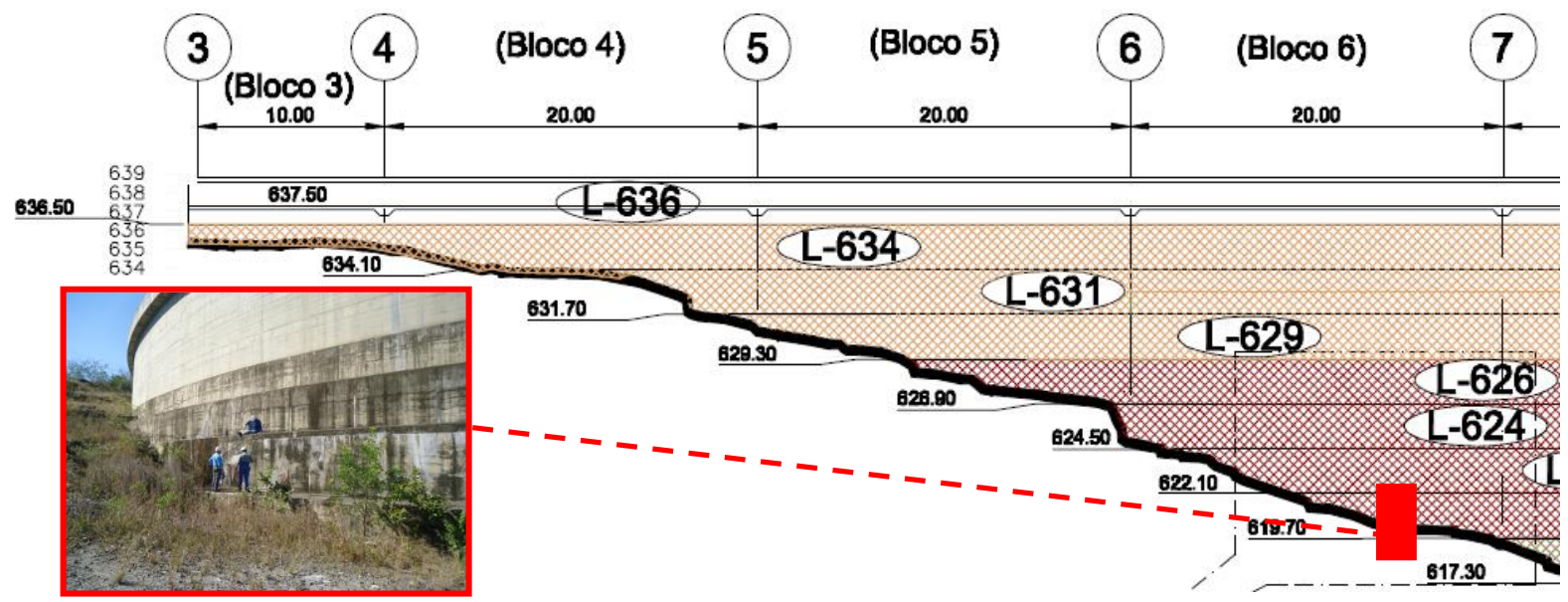

Figura 2: Local de extração de testemunhos de CCR em uma barragem localizada na região Sul do Brasil.

Os testemunhos analisados neste estudo foram identificados, para posterior realização de ensaios, conforme apresentado na Tabela 2. Além da obtenção dos testemunhos de CCR, foi obtido um testemunho de CCV, do contrapiso. Pela quantidade obtida, optou-se por apresentar os resultados somente para comparação e validação dos testes realizados com o CCR, haja vista que somente o ensaio de absorção é amplamente utilizado neste material.

Tabela 2: Identificação, descrição do local, cota e material do testemunho.

\begin{tabular}{ccc}
\hline Testemunhos & Cota (m) & Material \\
\hline 1.1327.16 - Face Jusante bl. 6/7 - Prof.: 0,00-0,28m & 622,1 & CCR \\
1.1326.16 A - Face Jusante bl. 6/7 - Prof.: 2,37-3,01m & 598,7 & CCR \\
1.1326.16 B - Face Jusante bl. 6/7 - Prof.: 2,37-3,01m & 598,7 & CCR \\
1.1330.16 - Face Jusante bl. 6/7 - Prof.: 0,00-0,77m & 622,1 & CCV \\
\hline
\end{tabular}

A dosagem de CCR desta região de análise teve um consumo de $80 \mathrm{~kg} / \mathrm{m}^{3}$ de cimento, fator a/c 1,69, e Fck especificado de $7 \mathrm{MPa}$, em 180 dias. O agregado utilizado foi identificado pela análise dos resultados das imagens de petrografia como sendo um diabásio com reatividade potencial. Pelo resultado da investigação qualitativa por petrografia, foram identificadas, além de outras fases químicas, a presença de sulfetos minerais, principalmente, a pirita $\left(\mathrm{FeS}_{2}\right)$ [16].

O equipamento utilizado para a retirada de testemunhos foi uma extratora da marca Hilti, modelo DD350, utilizando uma coroa diamantada com dimensões internas de $152 \mathrm{~mm}$ de diâmetro e $600 \mathrm{~mm}$ de comprimento da broca. O diâmetro recomendado pela norma NBR 7680 [17] é de 150 mm, definido em função da dimensão máxima 
MEDEIROS, B.L.; ET AL., AVALIAÇÃO DE TESTEMUNHOS DE CONCRETO COMPACTADO COM ROLO, COM INDÍCIOS DE ATAQUE POR SULFATO DE ORIGEM INTERNA, PELO ENSAIO DE PERMEABILIDADE AO AR - MÉTODO TORRENT. $2^{\circ}$ Simpósio

Paranaense de Patologia das Construções (20 SPPC), 2SPPC1023, pp. 275-288, 2017. DOI: 10.4322/2SPPC.2017.023

do agregado, de forma a não afetar os resultados mecânicos entre outros ensaios previstos para os testemunhos.

Para a realização dos ensaios de Torrent, foi utilizado o equipamento Torrent da marca Proceq. Também, foram utilizadas amostras de testemunhos de CCR e de CCV de $150 \mathrm{~mm}$ de diâmetro. As medidas foram efetuadas nas condições ambientais de laboratório de $(23 \pm 2){ }^{\circ} \mathrm{C}$ e em umidade relativa UR60\%. Como não há referências sobre os resultados do teste de Torrent em amostras de CCR, optou-se, nesta investigação, pela sua execução em ambos os lados dos testemunhos. A técnica de aplicação do CCR pode propiciar diferentes densidades entre as camadas de compactação, apresentando, assim, diferentes resultados.

Para a determinação da velocidade de propagação de onda em amostras de CCR, foram realizados os ensaios, segundo as diretrizes da NBR 8802 [18], coletando leituras longitudinais ao corpo de prova. A frequência de vibração utilizada foi de 54 kHz no equipamento Pundit PL 200 da marca Proceq.

O coeficiente de absorção capilar decorrente da ação de forças de atração dos poros do concreto sobre os líquidos que estão em contato com sua superfície foi obtido, segundo as recomendações da NBR 9779 [8].

O ensaio de absorção por imersão é considerado outro indicador da permeabilidade e foi desenvolvido segundo as recomendações da metodologia indicada pela NBR 9778 [9], no qual a absorção foi medida pelo processo de passagem da água por poros, em um material poroso como o concreto [6]. A avaliação final foi considerada em função dos limites normatizados, tais como: i) superiores a 6,3\% de absorção, considerados deficientes; ii) entre 4,2 e $6,3 \%$, considerados como normais e, iii) os valores menores que $4,2 \%$, como duráveis.

As investigações foram complementadas com a realização de análises com o microscópio eletrônico de varredura e a análise química elementar, em testemunho de CCR e de CCV, com o intuito de investigar possíveis indícios de reações expansivas devido à presença de minerais reativos. As microanálises foram realizadas em amostras de argamassas retiradas do interior do testemunho de concreto, em superfície de fratura e sem tratamento prévio (in natura). Foi utilizado um microscópio eletrônico de varredura, tipo FEG (field emission gun), modelo MIRA3 LM, marca TESCAN, com uso dos detectores de elétrons secundários (SE) e de elétrons retroespalhados (BSE) marca TESCAN. Os parâmetros do equipamento seguiram com a tensão de corrente do feixe de elétrons de $15 \mathrm{kV}$, intensidade do feixe (beam intensity) de 15,0, uma abertura do feixe (spot size) de $18,0 \mathrm{~nm}$, distância de trabalho (work distance-WD) de $15 \mathrm{~mm}$ e no modo de resolução do scanner.

A avaliação qualitativa e semiquantitativa dos elementos químicos presentes nos materiais, foi realizado a partir do uso do detector de espectrometria de raios- $X$, marca Oxford Instruments, sendo a investigação das fases químicas presentes pelo uso do software Aztec. 
MEDEIROS, B.L.; ET AL., AVALIAÇÃO DE TESTEMUNHOS DE CONCRETO COMPACTADO COM ROLO, COM INDÍCIOS DE ATAQUE POR SULFATO DE ORIGEM INTERNA, PELO ENSAIO DE PERMEABILIDADE AO AR - MÉTODO TORRENT. $2^{\circ}$ Simpósio Paranaense de Patologia das Construções (20 SPPC), 2SPPC1023, pp. 275-288, 2017. DOI: 10.4322/2SPPC.2017.023

\section{Resultados}

A permeabilidade ao ar pelo método Torrent [10] foi obtida a partir das leituras realizadas em ambos os lados do corpo de prova dos testemunhos analisados, denominados de L1 e L2. Ao realizar as leituras, foi identificada uma diferença de valores de kT e L, conforme pode ser verificado na Figura 3.
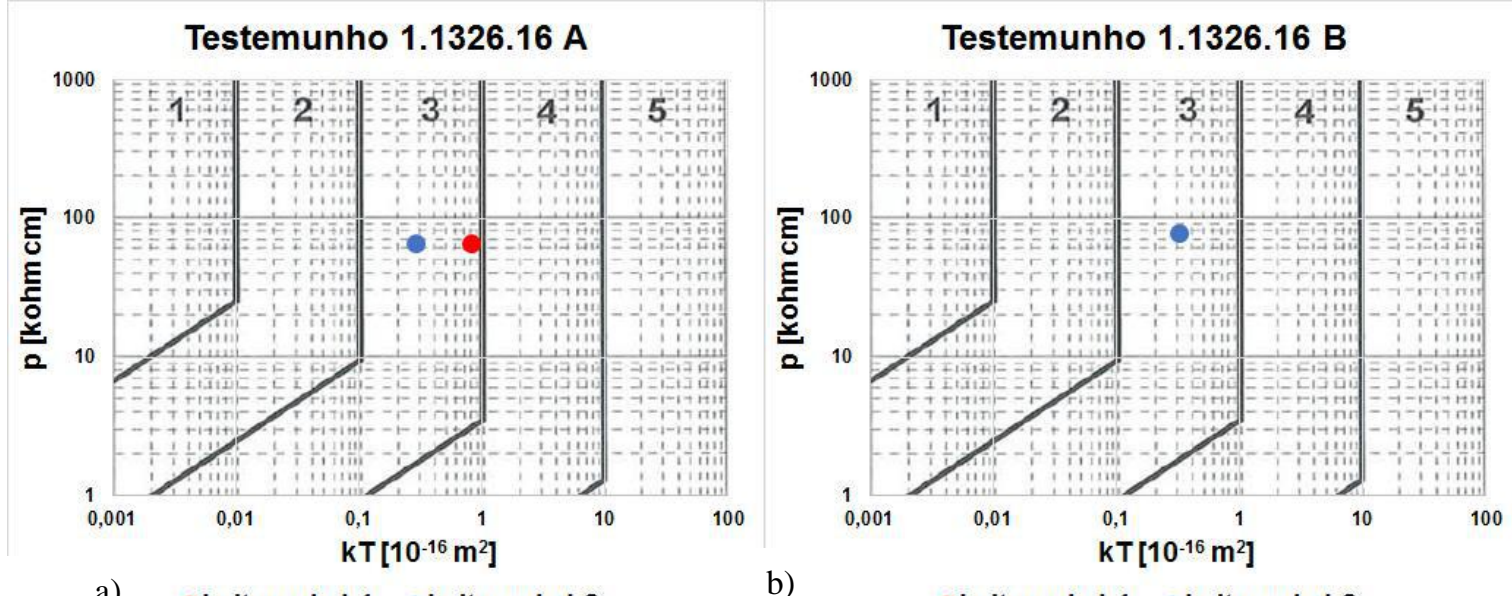

a)

- Leitura do L1 eleitura do L2

- Leitura do L1 - Leitura do L2

Figura 3: Gráficos resultantes das medidas de permeabilidade ao ar (kT) e da resistividade elétrica $\rho$ em dois lados ( $L 1$ e $L 2)$ dos testemunhos de CCR, 1.1326.16 $A(a)$ e1326.16 B(b).

O testemunho $1326.16 \mathrm{~A}$ apresentou, para a mesma resistividade, valores de $\mathrm{kT}$ diferentes, porém dentro da mesma faixa do índice 3 (concreto normal - Tabela 1). O testemunho identificado como 1.1326.16 B não possibilitou a leitura do L1, em função dos vazios apresentados no lado L1, conforme pode ser verificado em destaque pela seta na Figura 4. Estes vazios provavelmente foram provenientes do processo de compactação do CCR.

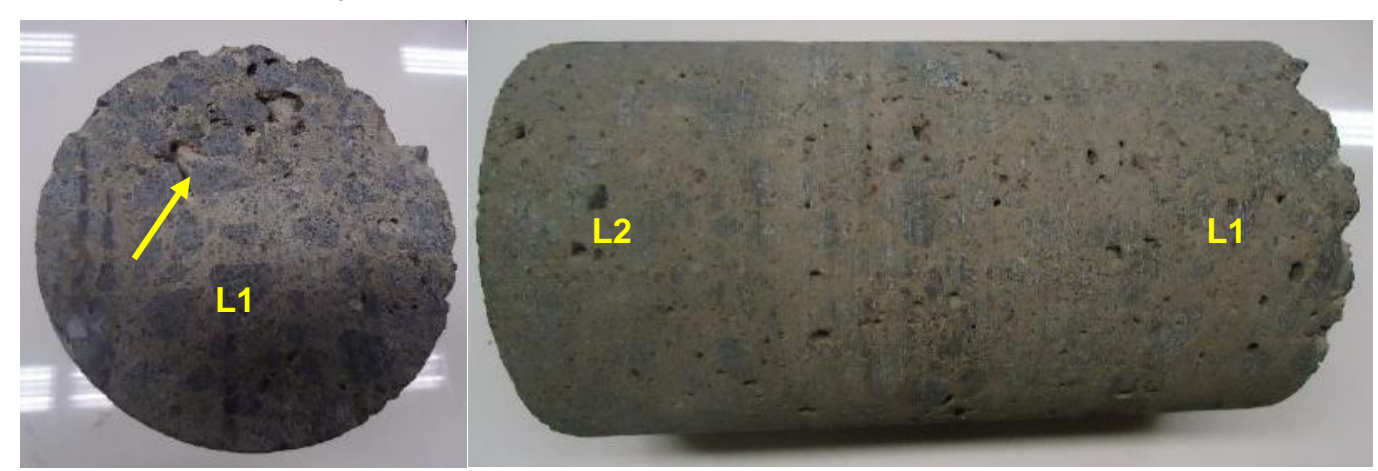

Figura 4: Vazios no lado L1 do testemunho 1.1326.16 B.

Para o testemunho de CCR (1.327.16), mais próximo do ambiente externo, ou seja ,mais superficial em relação à cota medida, os resultados obtidos para kT, Figura 5tanto para L1 quanto para L2 foram muito semelhantes, conforme mostrado na Figura 5(a), da faixa do índice 4, porém, próximos da faixa do índice 5, ou seja, de um concreto que pode ser classificado como ruim em relação aos demais testemunhos de CCR. 
MEDEIROS, B.L.; ET AL., AVALIAÇÃO DE TESTEMUNHOS DE CONCRETO COMPACTADO COM ROLO, COM INDÍCIOS DE ATAQUE POR SULFATO DE ORIGEM INTERNA, PELO ENSAIO DE PERMEABILIDADE AO AR - MÉTODO TORRENT. $2^{\circ}$ Simpósio

Paranaense de Patologia das Construções (20 SPPC), 2SPPC1023, pp. 275-288, 2017. DOI: 10.4322/2SPPC.2017.023

O testemunho de CCV (1.1330.16), (Figura 5b)) também próximo à superfície, apresentou diferença de leituras nos lados L1 e L2, porém ambos os resultados na mesma faixa do índice 4 (concreto ruim).

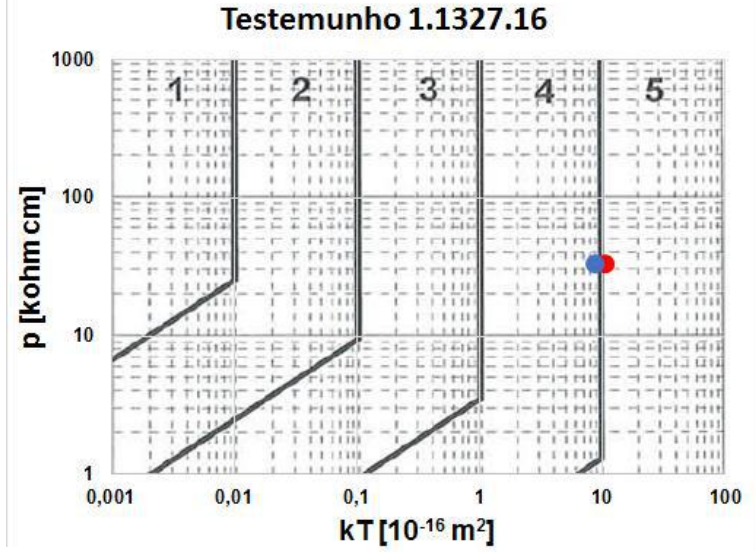

a) Leitura do L1 eleitura do L2

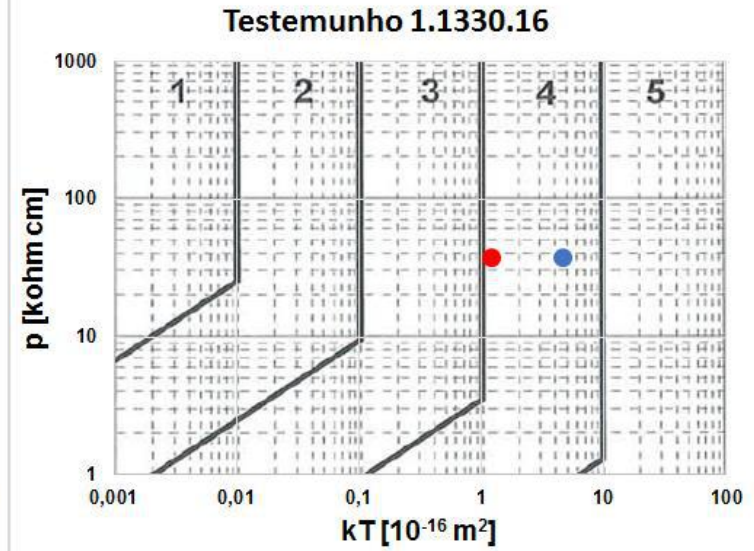

b) Leitura do L1 e Leitura do L2

Figura 5: Gráficos resultantes das medidas de permeabilidade ao ar (kT) e da resistividade elétrica $\rho$ em dois lados (L1 e L2) dos testemunhos de CCR 1.1327.16 (a) e de CCV - 1330.16 (b).

$\mathrm{Na}$ Tabela 3, estão apresentados os resultados obtidos nos ensaios de kT, da velocidade de propagação de onda, da resistividade ( $\rho)$, da absorção por capilaridade e por imersão, da média, do desvio padrão (DP) e do coeficiente de variação (CV) para os testemunhos de CCR e de CCV. Na Figura 6, estão indicados, na forma gráfica, os resultados dos ensaios ao longo das diferentes cotas da barragem.

Tabela 3: Resultados médios dos ensaios em testemunhos de CCR e CCV.

\begin{tabular}{c|c|c|c|c|c|c|c|c}
\hline & $\mathbf{k T}$ & $\mathbf{L}$ & $\begin{array}{c}\text { VEL. } \\
\text { DE } \\
\text { ONDA }\end{array}$ & $\mathbf{\rho}$ & \multicolumn{4}{|c}{ ABSORÇÃO } \\
\cline { 2 - 9 } $\begin{array}{c}\text { TESTEMUN } \\
\text { HO }\end{array}$ & & & & & $\begin{array}{c}\text { Capilar } \\
\text { idade }\end{array}$ & $\begin{array}{c}\text { Imersã } \\
\mathbf{0}\end{array}$ & $\begin{array}{c}\text { Índice } \\
\text { de } \\
\text { vazios } \\
(\%)\end{array}$ & $\begin{array}{c}\text { Massa } \\
\text { específi } \\
\mathbf{c a} \\
\left(\mathbf{k g} / \mathbf{d m}^{3}\right)\end{array}$ \\
\hline 1.1327 .16 & $1,04 \mathrm{E}-15$ & 0,10 & 3518 & 33,27 & 1,03 & 3,22 & 8,12 & 2,60 \\
\hline $1.1326 .16 \mathrm{~A}$ & $1,39 \mathrm{E}-15$ & 0,11 & 4075 & 66,00 & 0,60 & 2,81 & 6,80 & 2,49 \\
\hline $1.1326 .16 \mathrm{~B}$ & $3,29 \mathrm{E}-17$ & 0,04 & 4254 & 77,23 & 0,48 & 2,30 & 5,70 & 2,54 \\
\hline MÉDIA & $8,24 \mathrm{E}-16$ & 0,08 & 3949 & 58,83 & 0,70 & 2,78 & 6,87 & 2,54 \\
\hline DP & $5,78 \mathrm{E}-16$ & 0,03 & 313 & 18,65 & 0,24 & 0,38 & 0,99 & 0,04 \\
\hline CV & $70 \%$ & $38 \%$ & $8 \%$ & $32 \%$ & $34 \%$ & $13,55 \%$ & $14,39 \%$ & $1,77 \%$ \\
\hline 1.1330 .16 & $4,58 \mathrm{E}-16$ & 0,08 & 3252 & 37,53 & 2,13 & 6,20 & 15,04 & 2,58 \\
\hline
\end{tabular}


MEDEIROS, B.L.; ET AL., AVALIAÇÃO DE TESTEMUNHOS DE CONCRETO COMPACTADO COM ROLO, COM INDÍCIOS DE ATAQUE POR SULFATO DE ORIGEM INTERNA, PELO ENSAIO DE PERMEABILIDADE AO AR - MÉTODO TORRENT. $2^{\circ}$ Simpósio Paranaense de Patologia das Construções (20 SPPC), 2SPPC1023, pp. 275-288, 2017. DOI: 10.4322/2SPPC.2017.023

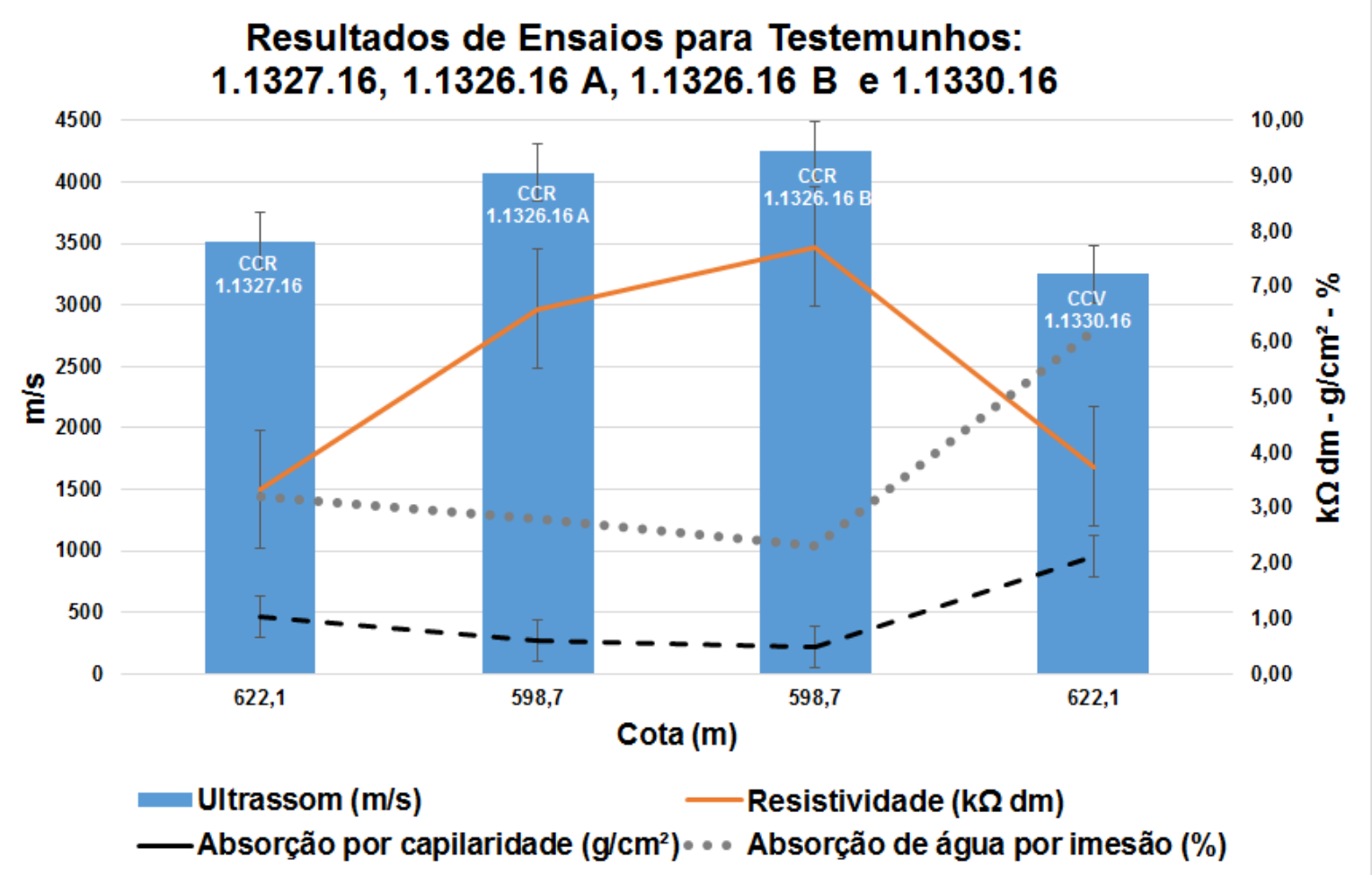

Figura 6: Apresentação gráfica dos resultados dos ensaios de resistividade, velocidade de onda e absorção por capilaridade e por imersão de testemunhos de CCR e CCV.

Pela análise dos dados da Figura 6, foi possível observar que, com o aumento da profundidade, ou seja, quanto mais distante da superfície, maior foi a resistividade e a velocidade de onda, com uma absorção por capilaridade e imersão baixa. Isto pode ser devido à oxigenação e umidade diferenciais em regiões superficiais, aos vazios presentes no material que não permitiram a interconexão dos poros capilares, mostrando valores baixos para este ensaio, bem como o possível preenchimento de poros com subprodutos. Sahuinco [6], relatou, em seus estudos, que a presença de íons agressivos no concreto aumenta a concentração iônica da solução aquosa dos poros e, assim, a sua condutividade elétrica, corroborando desta forma com os resultados obtidos para os testemunhos de CCR 1.1326.16 A e B.

Sengul [19], em seu estudo do uso da resistividade como indicador de durabilidade, apresenta valores acima de $35 \mathrm{k} \Omega$.cm como um concreto resistente a penetração por íons cloreto, o que está correlacionado com um material de menor porosidade. Desta forma, os testemunhos de CCR 1.1326.16 A e B teriam muito alta resistência a ataques por íons cloretos, e o CCR (1.1327.16) como o CCV (1.1330.16), teriam alta, o que pode estar correlacionado com a colmatação dos poros, devido à formação e deposição de subprodutos.

Para o testemunho de CCV (1.1330.16), foi possível observar um comportamento semelhante ao testemunho de CCR (1.1327.16) ambos situados na mesma cota da barragem, mesmo tendo este, propriedades diferenciadas em termos físicos, como a porosidade aparente. Hasparyk [20], obteve em seus estudos com concretos com maior consumo de cimento e degradados por RAA, resultados médios de 
MEDEIROS, B.L.; ET AL., AVALIAÇÃO DE TESTEMUNHOS DE CONCRETO COMPACTADO COM ROLO, COM INDÍCIOS DE ATAQUE POR SULFATO DE ORIGEM INTERNA, PELO ENSAIO DE PERMEABILIDADE AO AR - MÉTODO TORRENT. $2^{\circ}$ Simpósio

Paranaense de Patologia das Construções (20 SPPC), 2SPPC1023, pp. 275-288, 2017. DOI: 10.4322/2SPPC.2017.023

propagação da velocidade da onda entre 3080 e $2443 \mathrm{~m} / \mathrm{s}$, ou seja, inferiores aos obtidos neste trabalho, mesmo tendo sido observada a incidência de reações expansivas no concreto trabalhado.

Os resultados obtidos pelo ensaio de absorção ficaram entre 2,30\% e 3,22\%, sendo considerados baixos e condizentes com os resultados citados por Milani [21], onde se apresentou entre 3\% e 5\%. No trabalho realizado por Rezende et. al. [22], os valores de absorção obtidos em concreto de referência, foram obtidos entre $4 \%$ e $5 \%$. Ainda, foi verificado que valores de absorção abaixo de $4,2 \%$ e porosidade menor que $10 \%$ é qualificado como um concreto durável [6].

A massa especifica se manteve na média de $2,54 \mathrm{~kg} / \mathrm{dm}^{3}$, não havendo uma diminuição de densidade nos diferentes testemunhos de CCR. Sahuinco [6], apresentou índices de vazios médios de 13,56\% para CCV, onde pode-se dizer que o valor obtido com CCV apresentou resultado superior e o CCR apresentou um valor médio de $6,87 \%$.

Para o resultado do ensaio de absorção por capilaridade foi obtido um valor médio de $0,7 \mathrm{~g} / \mathrm{cm}^{2}$, abaixo do valor obtido em estudo realizado por Sahuinco [6], 1,21 $\mathrm{g} / \mathrm{cm}^{2}$, ou seja, o CCR apresentou um valor baixo baseado na porosidade.

As microanálises do concreto do testemunho de CCR foram efetuadas com o intuito de se identificar quaisquer indícios de reações expansivas, e assim ressaltar que os concretos analisados nos diferentes ensaios apresentaram em seus poros subprodutos de reações, ou seja, concretos com manifestações patológicas, alterando a característica inicial dos mesmos. A presença de subprodutos foi observada nas amostras de CCR $(1.1326 .16 \mathrm{~B})$, retiradas da cota $598,7 \mathrm{~m}$, conforme indicado nas micrografias da Figura 7 e da Figura 8 e validadas em suas análises químicas elementares.

A presença de sulfoaluminato de cálcio foi evidenciada na micrografia da Figura 7, bem como outros subprodutos de RAA conforme análises elementares realizadas nos pontos 1 e 2 indicados.

Para outra amostra de CCR analisada, foi possível identificar a presença de sulfoaluminatos de cálcio, tanto devido à presença de cristais com morfologia de agulhas aciculares, verificadas no destaque da micrografia, quanto pela análise elementar pontual, na região, conforme Figura 8. 
MEDEIROS, B.L.; ET AL., AVALIAÇÃO DE TESTEMUNHOS DE CONCRETO COMPACTADO COM ROLO, COM INDÍCIOS DE ATAQUE POR SULFATO DE ORIGEM INTERNA, PELO ENSAIO DE PERMEABILIDADE AO AR - MÉTODO TORRENT. $2^{\circ}$ Simpósio

Paranaense de Patologia das Construções (20 SPPC), 2SPPC1023, pp. 275-288, 2017. DOI: 10.4322/2SPPC.2017.023
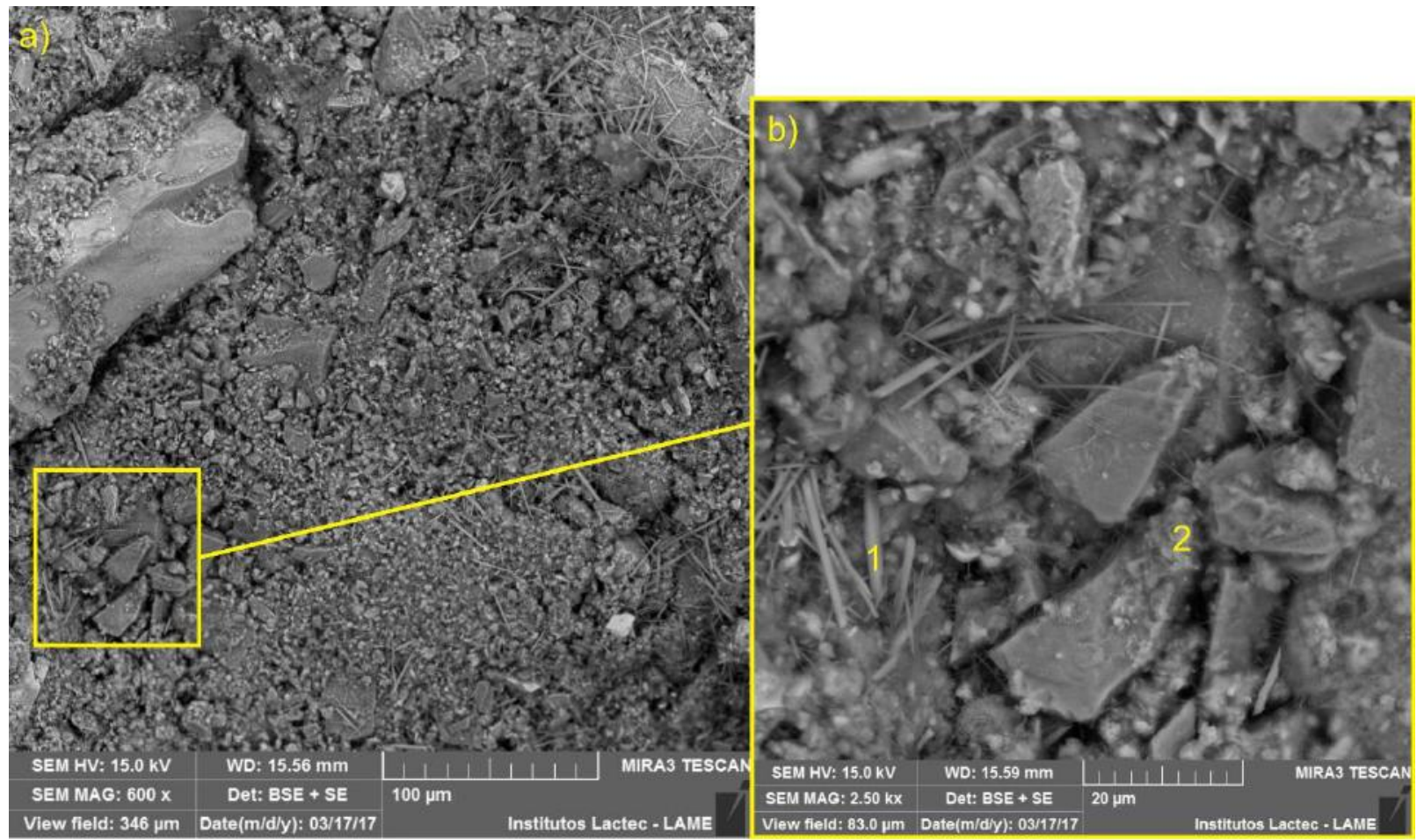

Figura 7: Micrografia da amostra retirada na cota 598,7m (1.1326.16 B), evidenciando o preenchimento dos poros com subprodutos (a); detalhe dos subprodutos formados (b).

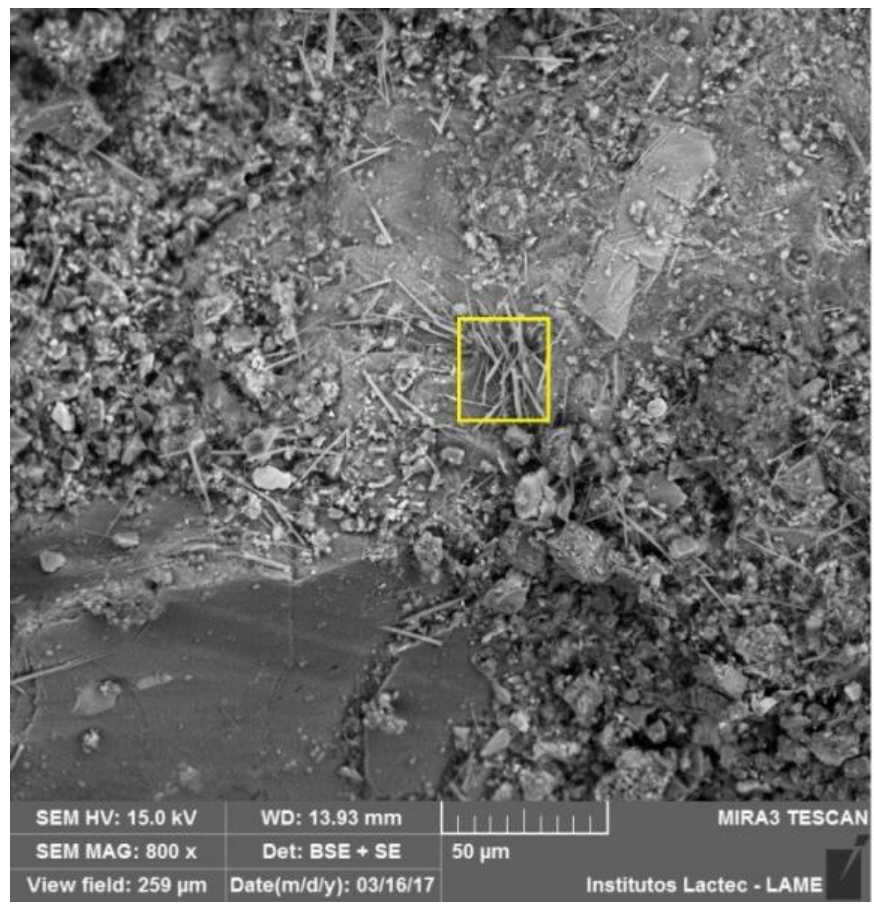

Figura 8: Micrografia da amostra retirada na cota 598,7m (1.1326.16 B), contendo subprodutos como o sulfoaluminato de cálcio, que pode ter característica expansiva.

Os resultados obtidos por MEV-EDS corroboraram com aqueles verificados pelas demais metodologias. Foi verificada a presença de subprodutos das reações de ASI e RAA, conforme apresentado na Figura 7 e na Figura 8, o que pode ter influenciado no aumento da resistividade e da velocidade de propagação de onda, devido à 
MEDEIROS, B.L.; ET AL., AVALIAÇÃO DE TESTEMUNHOS DE CONCRETO COMPACTADO COM ROLO, COM INDÍCIOS DE ATAQUE POR SULFATO DE ORIGEM INTERNA, PELO ENSAIO DE PERMEABILIDADE AO AR - MÉTODO TORRENT. $2^{\circ}$ Simpósio

Paranaense de Patologia das Construções (20 SPPC), 2SPPC1023, pp. 275-288, 2017. DOI: 10.4322/2SPPC.2017.023

presença de subprodutos no interior dos poros e vazios, sem a ocorrência aparente de microfissuração. No entanto, muito embora ainda não tenha sido verificada tal manifestação patológica, a ocorrência de poros, mesmo com a deposição de produtos expansivos, tendeu a manter a permeabilidade ao ar dos concretos em valores de ordem intermediária, estando os índices classificados como de normal a ruim.

\section{Conclusões}

Pelo ensaio de permeabilidade ao ar pelo método Torrent, realizado nos testemunhos de CCR, foram constatados resultados condizentes com os obtidos nos concretos analisados, indicando que o lado de leitura do ensaio pode estar alterado em função da compactação, da presença de vazios e de fissuras. Mesmo apresentando alto coeficiente de variação nas medidas em CCR, o que está de acordo com as propriedades do material, uma vez que estes apresentam maiores quantidades de vazios e compactação diferenciada entre as camadas, foi possível realizar repetições de leituras em cada lado dos testemunhos e verificar a semelhança de resultados obtidos pelo equipamento e em comparação às outras metodologias adotadas, balizando seu possível uso para tal material em laboratório.

A leitura da velocidade de onda obtida para o CCR foi superior à obtida para o CCV, sendo ambas condizentes com a de concretosde qualidade.

Os resultados de resistividade elétrica foram diferentes nos testemunhos de CCR, apresentando valores da ordem de $35 \mathrm{k} \Omega . \mathrm{cm}$ para as regiões de concretos próximos da superficie e de $75 \mathrm{k} \Omega . \mathrm{cm}$ para as regiões de mais elevada profundidade. Isso pode estar condizente com o grau de compactação, aeração e de disposição de sais e íons no interior dos poros. Esta mesma constatação, pode ser verificada no CCV próximo da superfície, com valores superiores a $35 \mathrm{k} \Omega . \mathrm{cm}$ (concreto resistente a ataques químicos [20]).

O elevado coeficiente de variação obtido nos diferentes ensaios pode estar condizente com as propriedades do CCR, pois estes apresentam maiores quantidade de vazios e compactação diferenciada nas camadas. Ainda, conforme verificado nas micrografias analisadas, os materiais apresentaram indícios de ASI, com uma composição química elementar indicativa de processos de RAA associados, tanto no CCR quanto no CCV. Esta constatação pode ter influenciado nas leituras nos ensaios, devido ao preenchimento de poros com produtos de reação.

\section{Agradecimentos}

Os autores agradecem à infraestrutura e ao apoio em recursos humanos e financiamento à COPEL GeT projeto P\&D 6491-0301/2013, à ANEEL, aos Institutos LACTEC, ao CNPq Lei 8010/90 (LI 15/2187214-1; LI 14/4695814-5; LI14/34107269), ao CNPq/PIBIT e de bolsa DT, processo 302672/2016-8*, e à Universidade Federal do Paraná - PPGECC/UFPR. 
MEDEIROS, B.L.; ET AL., AVALIAÇÃO DE TESTEMUNHOS DE CONCRETO COMPACTADO COM ROLO, COM INDÍCIOS DE ATAQUE POR SULFATO DE ORIGEM INTERNA, PELO ENSAIO DE PERMEABILIDADE AO AR - MÉTODO TORRENT. $2^{\circ}$ Simpósio Paranaense de Patologia das Construções (20 SPPC), 2SPPC1023, pp. 275-288, 2017. DOI: 10.4322/2SPPC.2017.023

\section{Referências}

[1] Campos, A.; López, C.M.; Aguado, A. (2015). Diffusion-reaction model for the internal sulfate attack in concrete. Construction and Building Materials, n.102, p.531-540.

[2] Melo, K.S. (2010). Estudo da formação de etringita tardia em concreto por calor de hidratação do cimento.Dissertação, Universidade federal de Goiás. Goiânia.

[3] Petaccia, G.; Lai, C. G.;Milazzo, C.; Natale, L. (2016) .The collapse of the Sella Zerbino Gravity dam. Engineering Geology. Pag 30-49.

[4] Zuffo, M. S. E R. (2005). Metodologia para avaliação de segurança de barragens. Universidade Estadual de Campinas. Campinas.

[5] Marques Filho, J. (2005). Maciço experimentais de laboratório de concreto compactado com rolo aplicado a barragens. Tese, Universidade Federal do Rio Grande do Sul, Porto Alegre, Brasil.

[6] Sahuinco, M. H. C. (2011). Utilização de métodos não destrutivos e semi destrutivos na avaliação de pontos de concreto. Dissertação. Escola Politécnica da Universidade de São Paulo. São Paulo.

[7] NBR 10786 (2013). Concreto endurecido - Determinação do coeficiente de permeabilidade à água. Associação Brasileira de Normas Técnicas - ABNT, Rio de Janeiro, Brasil.

[8] NBR 9779 (2012). Argamassa e concreto endurecidos - Determinação da absorção de água por capilaridade. Associação Brasileira de Normas Técnicas - ABNT, Rio de Janeiro, Brasil.

[9] NBR 9778 (2005). Argamassa e concreto endurecidos -Determinação da absorção de água, índice de vazios e massa específica. Associação Brasileira de Normas Técnicas - ABNT, Rio de Janeiro, Brasil.

[10] Torrent, R.; Frenzer, G. (1995). A method for the rapid determination of the coefficient of permeability of the "covercrete". Internacional symposium nondestrutive testing in civil engineering.

[11] Adámek, J; Juránková, V. (2010). Evaluation of durability of concrete by measurement of permeability for air and water. Modern building materials, structures and techniques. Lithuania.

[12] Ebensperger, L; Torrent, R. J. (2014). Ensayo in-situ de permeabilidad al aire: Un medidor de durabilidad? Cinpar, Santiago de Chile. Material e Structures.

[13] Neves, R.; Branco, F.; Brito, J. (2012). About the statistical interpretation of air permeability assessment results.

[14] Sentone, D. T. (2011). Desenvolvimento de método para medida de permeabilidade superficial de superfície de revestimento de argamassa. Dissertação, Universidade de São Paulo. São Paulo.

[15] Jacobs, F.; Hunkeler, F. (2006). Non destructive testing of the concrete cover evaluation of permeability test data. Internacional RILEM workshop, Madrid. 
MEDEIROS, B.L.; ET AL., AVALIAÇÃO DE TESTEMUNHOS DE CONCRETO COMPACTADO COM ROLO, COM INDÍCIOS DE ATAQUE POR SULFATO DE ORIGEM INTERNA, PELO ENSAIO DE PERMEABILIDADE AO AR - MÉTODO TORRENT. $2^{\circ}$ Simpósio Paranaense de Patologia das Construções (20 SPPC), 2SPPC1023, pp. 275-288, 2017. DOI: 10.4322/2SPPC.2017.023

[16] Oliveira, I. C. (2013). Análise de dados para elaboração de diretrizes visando à detecção de sulfetos e sulfatos na composição do CCR. Dissertação (Mestrado - Programa de Pós-Graduação em Engenharia de Construção Civil). Universidade Federal do Paraná. Curitiba.

[17] NBR 7680 (2015). Concreto - Extração, preparo ensaio e análise de testemunhos de estruturas de concreto. Parte 1: Resistencia à compressão axial. Associação Brasileira de Normas Técnicas - ABNT, Rio de Janeiro, Brasil.

[18] NBR 8802 (2013). Concreto - Determinação da velocidade de propagação de onda ultrassônica. Associação Brasileira de Normas Técnicas - ABNT, Rio de Janeiro, Brasil.

[19] Sengul, O. (2014). Use of electrical resistivity as an indicator for durability. Construction and building materials. Pag 434-441. Turkey.

[20] Hasparyk, N. P. (2005). Investigação de concretos afetados pela reação álcali agregado e caracterização avançada da exsudação. Tese de Doutorado em Engenharia Civil, Universidade Federal do Rio Grande do Sul, Porto Alegre.

[21] Milani, L. F. (2003). O uso do concreto compactado com rolo em barragens Tendencias futuras. Dissertação de mestrado em Engenharia de Energia. Universidade Federal de Itajubá. Itajubá.

[22] Rezende, M. L. S.; Nascimento, J. W. B.; Neves, G. A.; Silva, F. L. H.; Leal, A. F., Ferreira, H. B. (2012). Uso de metacaulim em concreto seco: uma abordagem por superfície de resposta. Ambiente Construido. Pag. 135-146. Porto Alegre. 